\title{
Neuropsychiatric Lupus with Antibody-Mediated Striatal Encephalitis
}

\author{
(D)B.P. Kelley, DJ.J. Corrigan, (D) S.C. Patel, and (D)B.D. Griffith
}

\begin{abstract}
SUMMARY: Systemic lupus erythematosus is a chronic autoimmune disease characterized by the production of autoantibodies resulting in tissue injury across multiple organs; up to $50 \%$ of patients develop neurologic involvement, collectively referred to as neuropsychiatric systemic lupus erythematosus. The cases in this clinical report will highlight a subtype of neuropsychiatric systemic lupus erythematosus demonstrating imaging findings of striatal inflammation responsive to plasmapheresis similar to those in the subset of $\mathrm{N}$-methyl-D-aspartate receptor autoimmune encephalitis that involves the striatum. Although the cause for this striking imaging appearance is not definitely known, literature will be presented supporting the hypothesis that it is due to peripheral anti-double-stranded DNA antibodies entering the central nervous system to cross-react with $N$-methyl-D-aspartate receptor antigens.
\end{abstract}

ABBREVIATIONS: ANA $=$ antinuclear antibody; dsDNA = double-stranded DNA; NMDAr $=$ N-methyl-D-aspartate receptor; NPSLE $=$ neuropsychiatric systemic lupus erythematosus; $S L E=$ systemic lupus erythematosus

S ystemic lupus erythematosus (SLE) is a chronic systemic autoimmune disorder with an overall prevalence of 50 in 100,000 that most often affects women of child-bearing age and has an unpredictable clinical course characterized by periods of remission and acute flares. ${ }^{1}$ Lupus can affect almost any organ system in the body but often has neuropsychiatric symptoms that still remain poorly understood despite being one of the most common manifestations of the disease. ${ }^{2}$ The general term "neuropsychiatric systemic lupus erythematosus" (NPSLE) refers to any form of lupus with neuropsychiatric symptoms that are thought to be related to this systemic autoimmune condition. ${ }^{2}$ NPSLE may be the first manifestation of SLE and can be seen in up to $50 \%$ of patients with lupus, but there is wide variation in the reported prevalence of NPSLE in lupus among adults (14\%-80\%) and children $(22 \%-95 \%))^{2,3}$

NPSLE has been associated with a number of specific autoantibodies, and while vascular complications of SLE resulting in acute ischemic stroke or dural venous sinus thrombosis are gen-

Received June 14, 2018; accepted after revision July 25.

From the Department of Neuroradiology, Henry Ford Hospital, Detroit, Michigan.

Please address correspondence to Brendan P. Kelley, MD, MSc, Department of

Radiology, Henry Ford Hospital, 2799 W Grand Blvd, Detroit, MI 48202;

e-mail: brendank@rad.hfh.edu; @brendanpkelley; @brentdgriffith

三 Indicates article with supplemental on-line tables.

Indicates article with supplemental on-line photos.

http://dx.doi.org/10.3174/ajnr.A5842 erally considered to have the closest association with antibodies related to antiphospholipid antibody syndrome, ${ }^{4}$ it remains unclear whether other antibody subtypes such as those targeting double-stranded DNA (dsDNA) or ribosomal P antigens are more closely associated with the development of NPSLE. ${ }^{1,2}$ AntidsDNA antibodies are an antinuclear antibody (ANA) subtype that targets antigens within the dsDNA and represent one of the most specific serum autoantibodies in SLE. ${ }^{3,5,6}$ Although not widely considered clinically, there is growing support in the literature for a form of autoimmune encephalitis in patients with lupus who present with neuropsychiatric symptoms caused by peripheral anti-dsDNA antibodies entering the central nervous system to cross-react with $N$-methyl-D-aspartate receptor (NMDAr) antigens. ${ }^{2,4-7}$ While certain aspects of this process remain unclear, such as whether peripheral dsDNA antibodies enter the CNS via the choroid plexus ${ }^{8}$ or by crossing a compromised blood-brain barrier, ${ }^{5}$ there is a consensus that on entering the CNS, cross-reactivity of anti-dsDNA antibodies with NMDAr antigens mediates a nonthrombotic and nonvasculitic pathology in NPSLE with features of neuronal excitotoxicity. ${ }^{4-8}$

Many of the functions of the striatum are facilitated by glutaminergic and dopaminergic input, ${ }^{9}$ and this may partially explain why anti-NMDAr and anti-dopamine D2 receptor antibodies are associated with this form of autoimmune encephalitis via targeting these antigens in the striatum. ${ }^{4,6,10}$ Anti-NMDAr encephalitis was the first form of autoimmune encephalitis described by Dalmau et al in $2007^{11}$ and is now an established clinical diagnosis, 


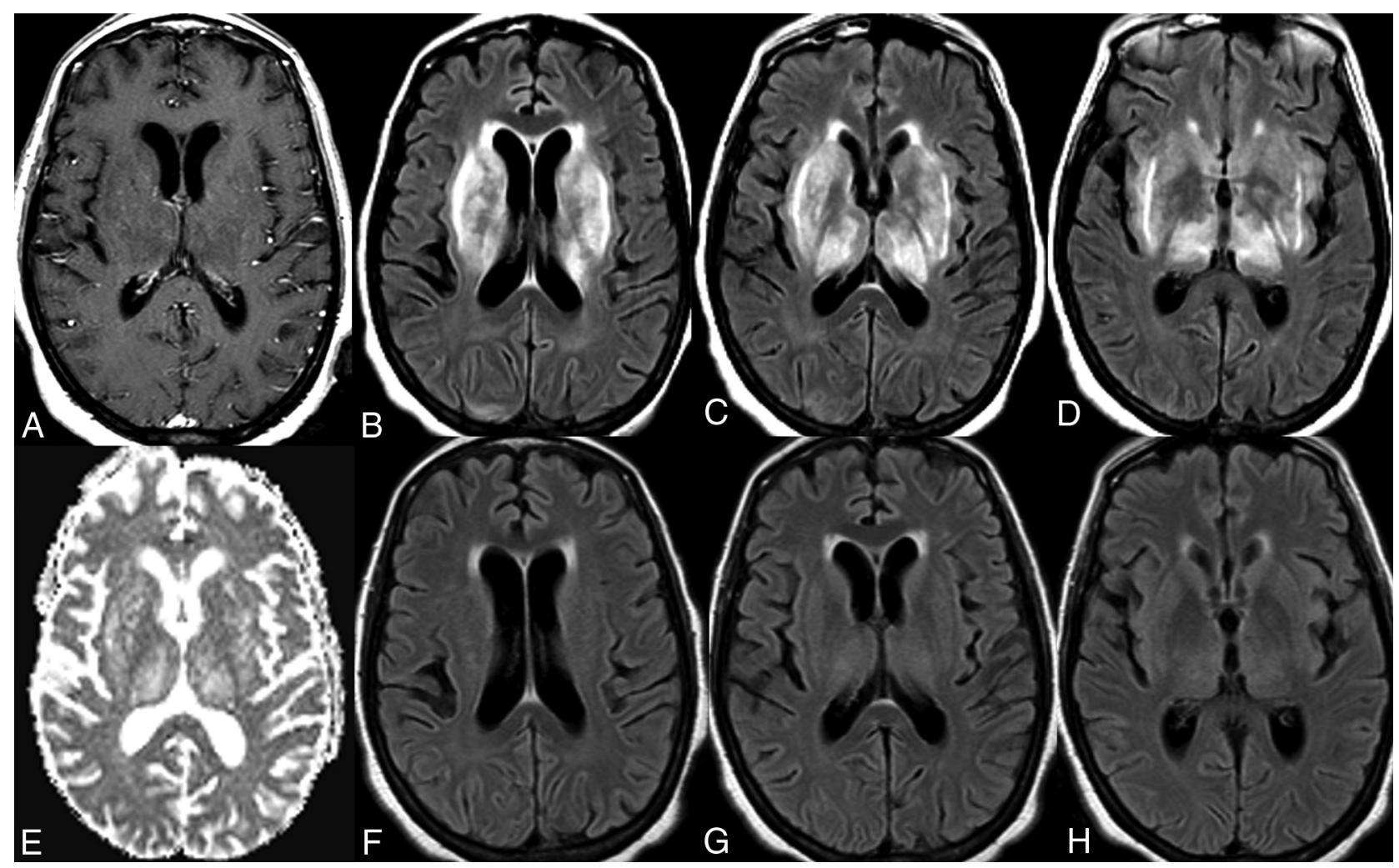

FIG 1. Neuropsychiatric lupus with antibody-mediated striatal encephalitis. A 68-year-old woman with a medical history of rheumatoid arthritis, systemic lupus erythematosus, type 2 diabetes mellitus, and hypertension presents with cognitive decline and generalized weakness. MR imaging of the brain demonstrates bilateral symmetric T2/FLAIR hyperintensity of the basal ganglia, thalami, and surrounding white matter ( $B-D$ ) without restricted diffusion $(E)$ or postcontrast enhancement $(A)$. An IV steroid regimen was initiated but stopped due to gastrointestinal bleeding, and the patient received 5 rounds of plasmapheresis with a positive clinical response. Follow-up MR imaging of the brain demonstrates near-complete resolution of previous T2/FLAIR signal abnormality $(F-H)$.

with subsequent discovery of many other antibodies responsible for various other forms of autoimmune encephalitis. ${ }^{10,12}$ Autoimmune encephalitis of the striatum is less common than other subtypes, only reported in $8 \%$ of anti-NMDAr cases in one of the largest studies conducted to date. ${ }^{13}$ Similarly, antibody-mediated inflammation of the striatum in SLE is also relatively uncommon, with basal ganglia involvement only reported in $7 \%$ of patients with lupus with neuropsychiatric symptoms. $^{14}$

Antibody-mediated neuroinflammation often presents with nonspecific clinical features, which can lead to a delay in the diagnosis and treatment, but fortunately, these patients may respond favorably, in the absence of an underlying malignancy, to early intervention consisting of immunosuppression with pulse dose corticosteroids, plasmapheresis to remove the circulating antibodies, and long-term management with steroid-sparing monoclonal therapies capable of B-cell depletion such as rituximab. ${ }^{15-17}$ Antibody-mediated striatal inflammation in NPSLE is no exception and demonstrates similar clinical presentations, MRI findings, and early response to treatment as traditional autoimmune striatal encephalitis caused by anti-NMDAr antibodies. ${ }^{16,18,19}$

This clinical report emphasizes the MR imaging brain findings and response to plasmapheresis in patients with SLE with antibody-mediated striatal encephalitis in the setting of anti-dsDNA antibodies. This retrospective study was approved by the institutional review board.

\section{Case Series}

Unless otherwise specified, all patients were afebrile and hemodynamically stable on presentation with a negative toxometabolic/ infectious work-up, positive serum anti-dsDNA antibodies, and CSF analysis consistent with mild-to-moderate lymphocytic pleocytosis (defined as 10-100 white blood cells/ $\mu \mathrm{L}$ and protein, $50-$ $150 \mathrm{mg} / \mathrm{dL})$.

\section{Case 1}

A 68-year-old woman with a medical history of rheumatoid arthritis, SLE, and type 2 diabetes mellitus presented with cognitive decline and generalized weakness. MR imaging of the brain demonstrated bilateral symmetric T2/FLAIR hyperintensity of the basal ganglia, thalami, and surrounding white matter without restricted diffusion or postcontrast enhancement (Fig $1 A-E$ ). An IV steroid regimen was attempted twice (methylprednisolone on both occasions) but was discontinued due to the complication of gastrointestinal bleeding. She was ultimately given the clinical diagnosis of autoimmune encephalitis and received 5 sessions of plasmapheresis with prompt clinical and radiologic improvement. Follow-up MR imaging of the brain demonstrated near-complete resolution of the previous T2/ FLAIR signal abnormality (Fig $1 F-H)$.

\section{Case 2}

A 20-year-old woman with a medical history of SLE presented with worsening headaches, anxiety, and cognitive impairment. 


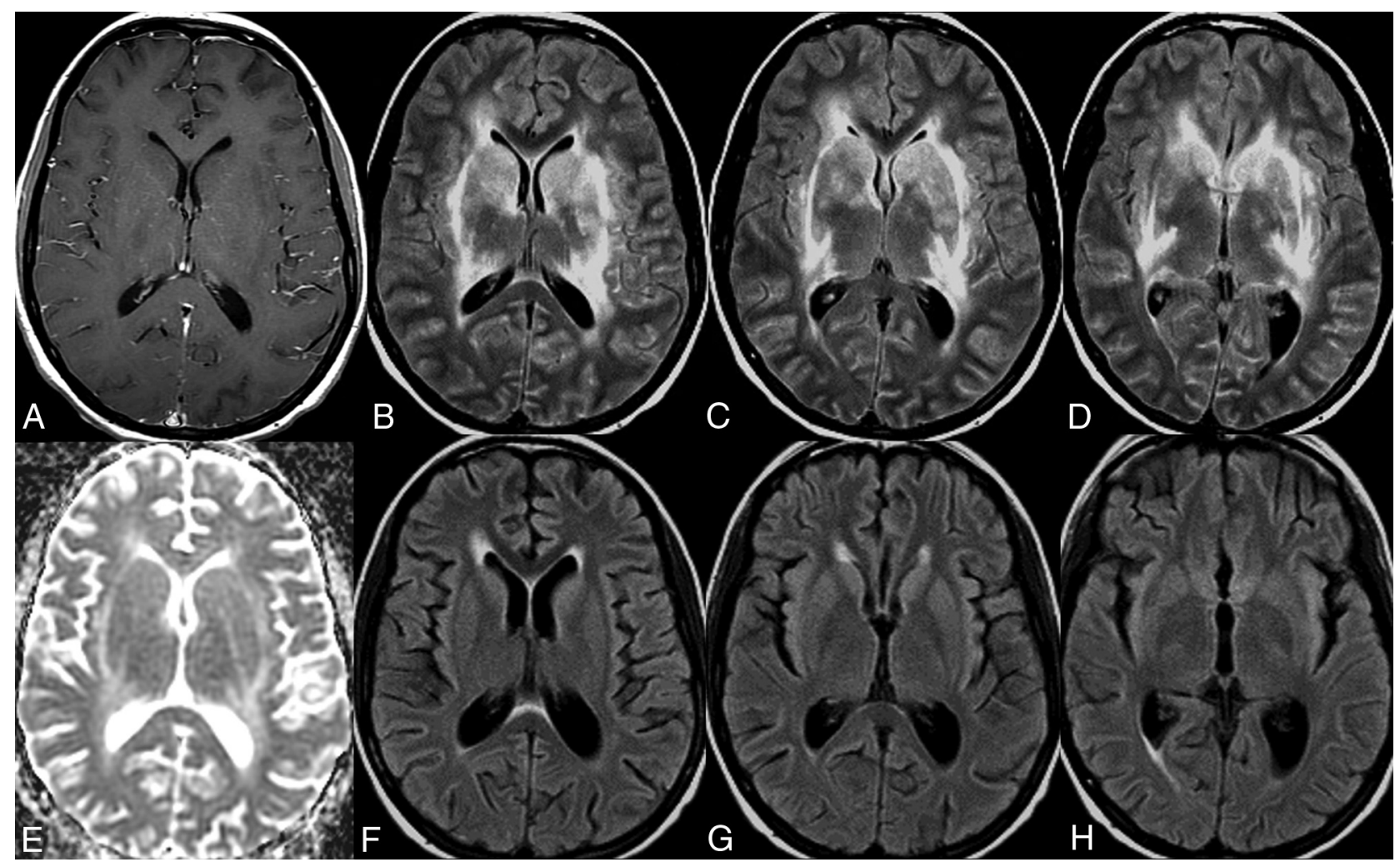

FIG 2. Neuropsychiatric lupus with antibody-mediated striatal encephalitis. A 20-year-old woman with a medical history of systemic lupus erythematosus, depression, and anxiety disorder presents with progressively worsening headaches, agitation, cognitive dysfunction, arthralgia, fever, and nausea. MR imaging of the brain demonstrates bilateral symmetric T2/FLAIR hyperintensity of the basal ganglia and surrounding white matter $(B-D)$ without restricted diffusion $(E)$ or postcontrast enhancement $(A)$. The patient did not experience much improvement on oral or IV steroids but had a good clinical response to 6 sessions of plasmapheresis. Follow-up MR imaging of the brain demonstrates near-complete resolution of previous T2/FLAIR signal abnormality with interval development of generalized mild brain atrophy $(F-H)$.

She was on levetiracetam and phenytoin after 1 episode of seizure that was thought to be secondary to lupus cerebritis. MR imaging brain examination demonstrated bilateral symmetric T2/FLAIR hyperintensity of the basal ganglia and surrounding white matter without restricted diffusion or postcontrast enhancement (Fig 2A-E). She did not experience much improvement on an oral/IV steroid regimen or cyclophosphamide infusion and experienced a breakthrough seizure during her inpatient work-up. She was ultimately given the clinical diagnosis of autoimmune encephalitis and received 6 rounds of plasmapheresis with clinical and radiologic improvement. Follow-up MR brain imaging demonstrated nearcomplete resolution of the previous T2/FLAIR signal abnormality with interval development of generalized mild brain atrophy (Fig $2 F-H$ ).

\section{Case 3}

A 24-year-old woman with a recent diagnosis of SLE presented with expressive aphasia and headaches. The MR imaging brain examination demonstrated bilateral symmetric T2/FLAIR hyperintensity of the caudate and, to a lesser degree, the lentiform nuclei, with patchy T2/FLAIR hyperintensity of the left greater-thanright thalami without restricted diffusion or postcontrast enhancement (Fig $3 A-E$ ). The patient experienced mild gradual improvement on IV steroid therapy with mild improvement in
T2/FLAIR signal abnormality on the 10-day follow-up MR imaging of the brain (Fig $3 F-H)$.

\section{Case 4}

A 20-year-old man with a medical history of SLE and dilated cardiomyopathy presented with altered mental status. An MR imaging brain examination demonstrated diffuse cortical atrophy, bilateral symmetric T1 and T2/FLAIR hyperintensity of the basal ganglia, and scattered small foci of T2/FLAIR hyperintensity in the bilateral supratentorial white matter without restricted diffusion or suspicious postcontrast enhancement (Fig 4). The patient ultimately died of cardiac arrest. The postmortem examination revealed lupus-associated vasculitis in the small-caliber arteries of the brain with transmural infiltrates of polymorphonuclear inflammatory cells with areas of destruction of the elastic lamina and fibrosis of the intima and media. It was noted that the basal ganglia only demonstrated a few blood vessels with transmural inflammation but showed geographic areas of parenchymal necrosis with dystrophic calcification, infiltrates of foamy macrophages, gliosis, and rarefaction. Cortical neurons of the cerebrum, brain stem, and spinal cord were well-preserved and within normal limits.

\section{Case 5}

A 37-year-old man with a medical history of SLE presented to an outside hospital after a flulike episode with altered mental

AJNR Am J Neuroradiol 39:2263-69 Dec 2018 www.ajnr.org 


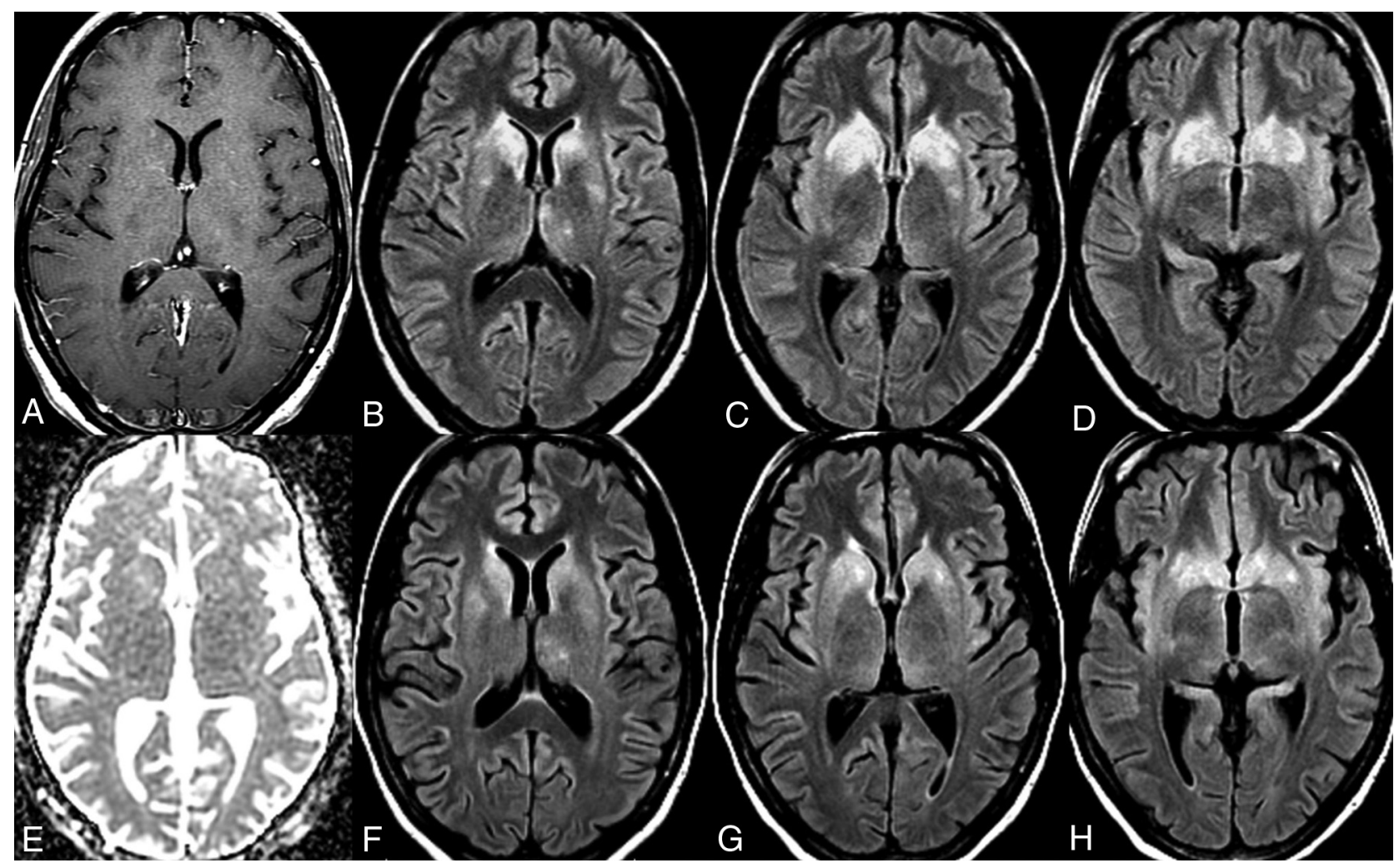

FIG 3. Neuropsychiatric lupus with antibody-mediated striatal encephalitis. A 24-year-old woman with a recent diagnosis of systemic lupus erythematosus on daily oral prednisone presents with discoid rash, aphthous oral ulcers, dysarthria, expressive aphasia, headaches, and vomiting. MR imaging of the brain demonstrates bilateral symmetric T2/FLAIR hyperintensity of the caudate and, to a lesser degree, the lentiform nuclei, with patchy T2/FLAIR hyperintensity of the left-greater-than-right thalami $(B-D)$ without restricted diffusion $(E)$ or postcontrast enhancement $(A)$. The patient was treated with a 7-day course of IV steroid therapy without plasmapheresis and experienced mild gradual clinical improvement. Follow-up MR imaging of the brain demonstrates mild reduction in T2/FLAIR hyperintensity $(F-H)$.

status, emesis, headaches, and dystonia, but he was transferred to our institution after development of refractory seizure and multisystem organ failure. An initial noncontrast MR imaging brain examination at the outside institution demonstrated extensive bilateral areas of restricted diffusion and T2/FLAIR hyperintensity throughout the supratentorial and infratentorial white matter with bilateral deep gray T2/FLAIR hyperintensity without restricted diffusion in the bilateral caudate, putamen, and thalamus (Fig $5 A-F$ ). Right frontal brain biopsy was also performed and revealed diffuse reactive astrocytosis with mild microglial activation, minimal perivascular lymphocytic infiltrates, and an unremarkable appearance of the parenchymal blood vessels of the cerebral cortex and white matter without features of spongiform encephalopathy or an infectious process. He was given the diagnosis of autoimmune lupus cerebritis with seizures and received 3 days of pulse dose IV steroids with prednisone taper. Serial MR imaging brain examinations during the 6-week inpatient admission demonstrated a progressive decrease in the T2/FLAIR signal abnormality. Outpatient 3-month follow-up MR imaging of the brain demonstrated decreased T2/FLAIR hyperintensity but interval development of prominent atrophy and intrinsic T1 hyperintensity in regions of previous signal abnormality (Fig $5 G$ ). His clinical follow-up was consistent with improved inflammation but severe chronic morbidity after this monophasic episode.

\section{Companion Case (Anti-NMDAr Encephalitis)}

A 38-year-old man presented with progressively worsening cognitive impairment, orofacial dyskinesia, and bilateral upper extremity chorea. MR imaging of the brain demonstrated bilateral symmetric T2/FLAIR hyperintensity of the dorsal striatum (caudate and lentiform nucleus) without restricted diffusion or postcontrast enhancement (Fig $6 A-C$ and $E$ ). The autoimmune work-up was extensive but was initially negative for serum autoantibodies, including anti-dsDNA antibodies. He underwent a 3 -day pulse IV steroid regimen with prednisone taper that resulted in gradual symptom improvement during 2-3 weeks but with persistent striatal T2/FLAIR abnormalities on the follow-up MR imaging of the brain, which also demonstrated interval development of some intrinsic T1 hyperintensity in the left striatum (Fig 6D). He was initially transferred to subacute rehabilitation but was readmitted for plasmapheresis when serum antibody testing was positive for anti-NMDAr antibodies. He completed 5 rounds of plasmapheresis with a good response. Follow-up MR imaging of the brain demonstrated reduced T2/FLAIR signal abnormality with atrophy of the caudate heads and persistent left striatal T1 hyperintensity (Fig $6 H)$. Note that this patient did not have SLE or anti-dsDNA antibodies but instead had positive NMDAr antibodies with a similar therapeutic response to a treatment regimen tailored for autoimmune encephalitis. 


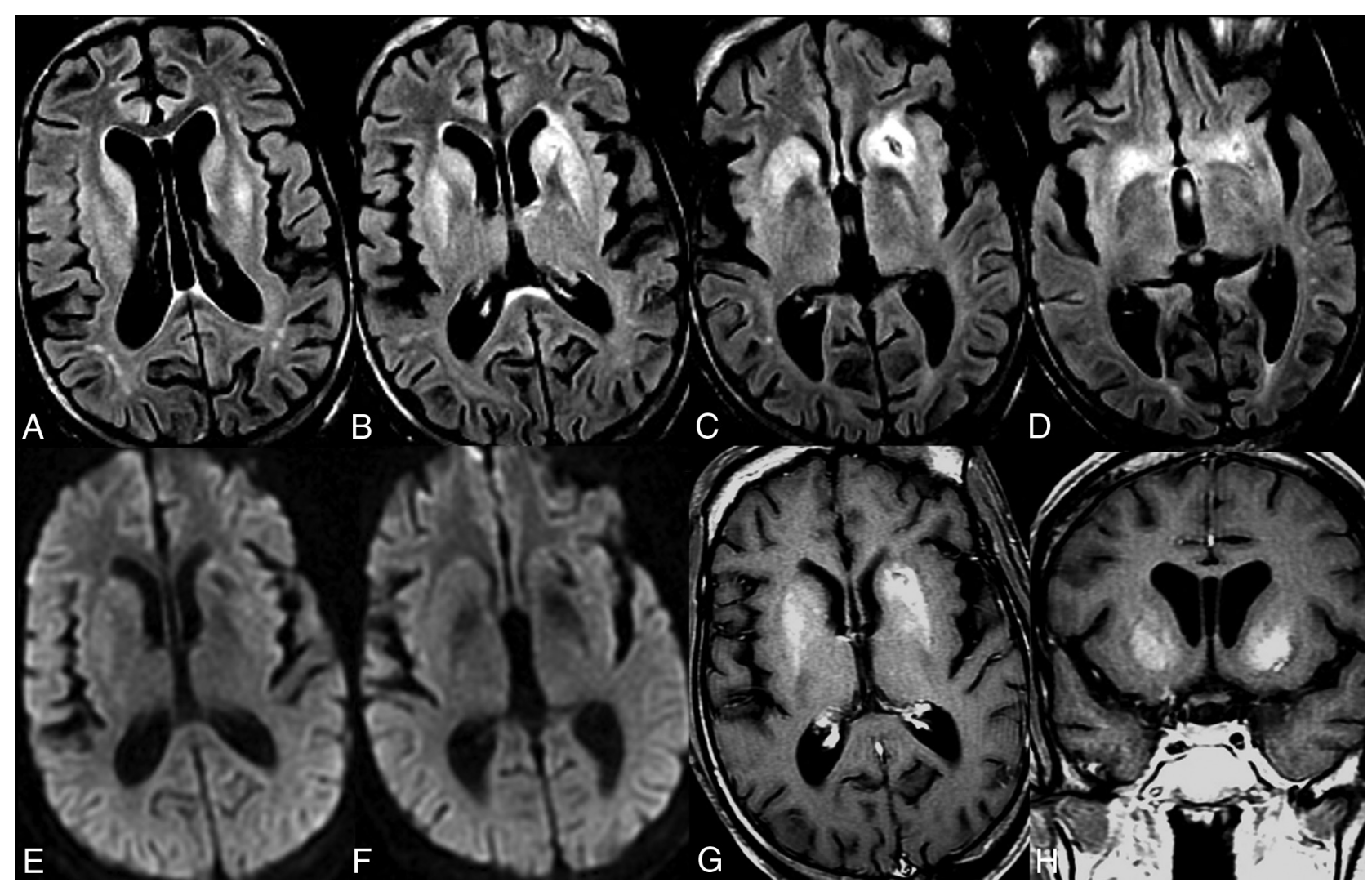

FIG 4. Neuropsychiatric lupus with antibody-mediated striatal encephalitis. A 20-year-old man with a medical history of systemic lupus erythematosus, paroxysmal atrial fibrillation, and dilated cardiomyopathy presents with altered mental status. MR imaging of the brain demonstrates advanced generalized brain atrophy with scattered bilateral frontoparietal T2/FLAIR white matter hyperintensities $(A$ and $B)$ and bilateral symmetric striatal abnormalities characterized by T2/FLAIR hyperintensity $(A-D)$ and T1 hyperintensity $(G$ and $H)$ without postcontrast enhancement or restricted diffusion but blooming artifacts in the left striatum from hemosiderin deposition ( $E$ and $F$ ). The patient developed refractory heart failure and ultimately died of cardiac arrest. Note the intrinsic basal ganglionic T1 hyperintensity correlated with coagulative necrosis on brain postmortem examination.

\section{DISCUSSION}

All forms of antibody-mediated disease can be defined at the most basic level by a loss of tolerance to self that results in immunemediated inflammation of target tissues. ${ }^{6,10,20}$ In patients with SLE, the loss of immune tolerance to autoantigens within the body stimulates the clonal expansion of selective B-cell populations that produce specific autoantibodies that are released into the circulation to eventually bind to their target and initiate an inflammatory immune response, which often has features of immune complex deposition, complement activation, inflammatory cytokine production, or local recruitment of macrophages. ${ }^{2,4}$ Despite the diversity of brain pathology that can occur in lupus, which is reflected in the wide range of MR imaging findings, ${ }^{14}$ the presence of bilateral symmetric T2/FLAIR hyperintense signal changes within the caudate and putamen without evidence of restricted diffusion or postcontrast enhancement represents a unique neuroimaging pattern that, in the appropriate clinical setting, is highly suggestive of autoimmune encephalitis of the striatum. Note also that in some of the presented cases, particularly those with the worst outcomes, intrinsic T1 hyperintensity was also seen within the basal ganglia, perhaps reflecting the development of coagulative necrosis in the setting of prolonged antibodymediated inflammation and excitatory glutamate neurotoxicity, suggesting that this finding may represent a poor prognostic feature (Figs 4-6).
While it is clear that prompt diagnosis and treatment of autoimmune encephalitis is associated with improved clinical outcomes, establishing the diagnosis first requires exclusion of many more common causes of altered mental status, such as stroke, intracranial hemorrhage, trauma, infection, or toxometabolic encephalopathy ${ }^{15,20}$ Neuroimaging plays an important role in this diagnostic work-up, and characteristic MR imaging brain findings of bilateral symmetric T2/FLAIR hyperintense signal changes within the caudate and putamen without restricted diffusion or postcontrast enhancement may be the first indication that autoimmune striatal encephalitis should be considered. ${ }^{10,19,21}$ Please see On-line Table 1 and On-line Figs 1-6 for a comprehensive list of diagnostic considerations in the setting of bilateral symmetric T2/FLAIR hyperintense signal changes within the caudate and putamen, ${ }^{22,23}$ as well as On-line Table 2 for suggested clinical and laboratory tests in patients with suspected autoimmune striatal encephalitis. ${ }^{15,20}$

Maximizing clinical outcomes in these patients requires a multidisciplinary approach that relies on a combination of clinical, laboratory, and imaging data. The neuroimaging findings in these patients are quite striking, and though they are nonspecific, many of the other etiologies on the differential diagnosis can be excluded during the diagnostic work-up. Similar to autoimmune encephalitis, it is important to emphasize that positive antibody testing within serum or cerebrospinal AJNR Am J Neuroradiol 39:2263-69 Dec 2018 www.ajnr.org 


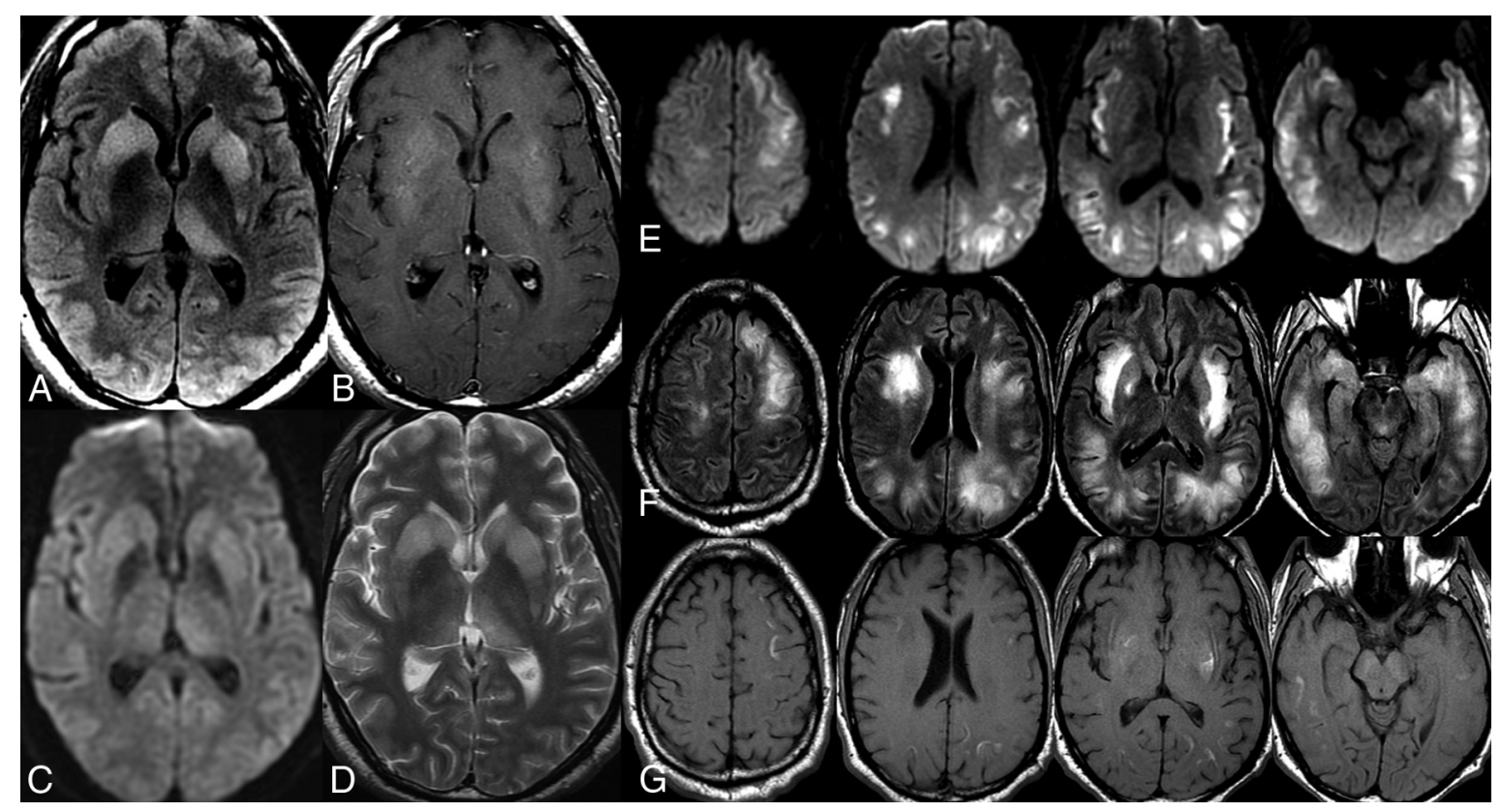

FIG 5. Neuropsychiatric lupus with antibody-mediated striatal encephalitis. A 37-year-old man develops encephalopathy and refractory seizures following flulike symptoms with subsequent development of multisystem organ failure. MR imaging of the brain demonstrates a diffuse extensive burden of restricted diffusion $(E)$ and T2/FLAIR hyperintensity $(F)$ in the supratentorial white matter with deep gray T2/FLAIR hyperintensity $(A$ and $D)$ without restricted diffusion $(C)$ in the bilateral caudate, putamen, and thalami. The patient received supportive care in the intensive care unit with a 3-day course of IV steroids and prednisone taper without seizure recurrence. Serial MR imaging brain examinations demonstrated gradual resolution of restricted diffusion with a mild progressive decrease in both gray and white matter T2/FLAIR hyperintensity (not shown) but with progressive development of intrinsic $T$ hyperintensity within the subcortical white matter $(G)$ and basal ganglia $(B)$, consistent with coagulative necrosis.

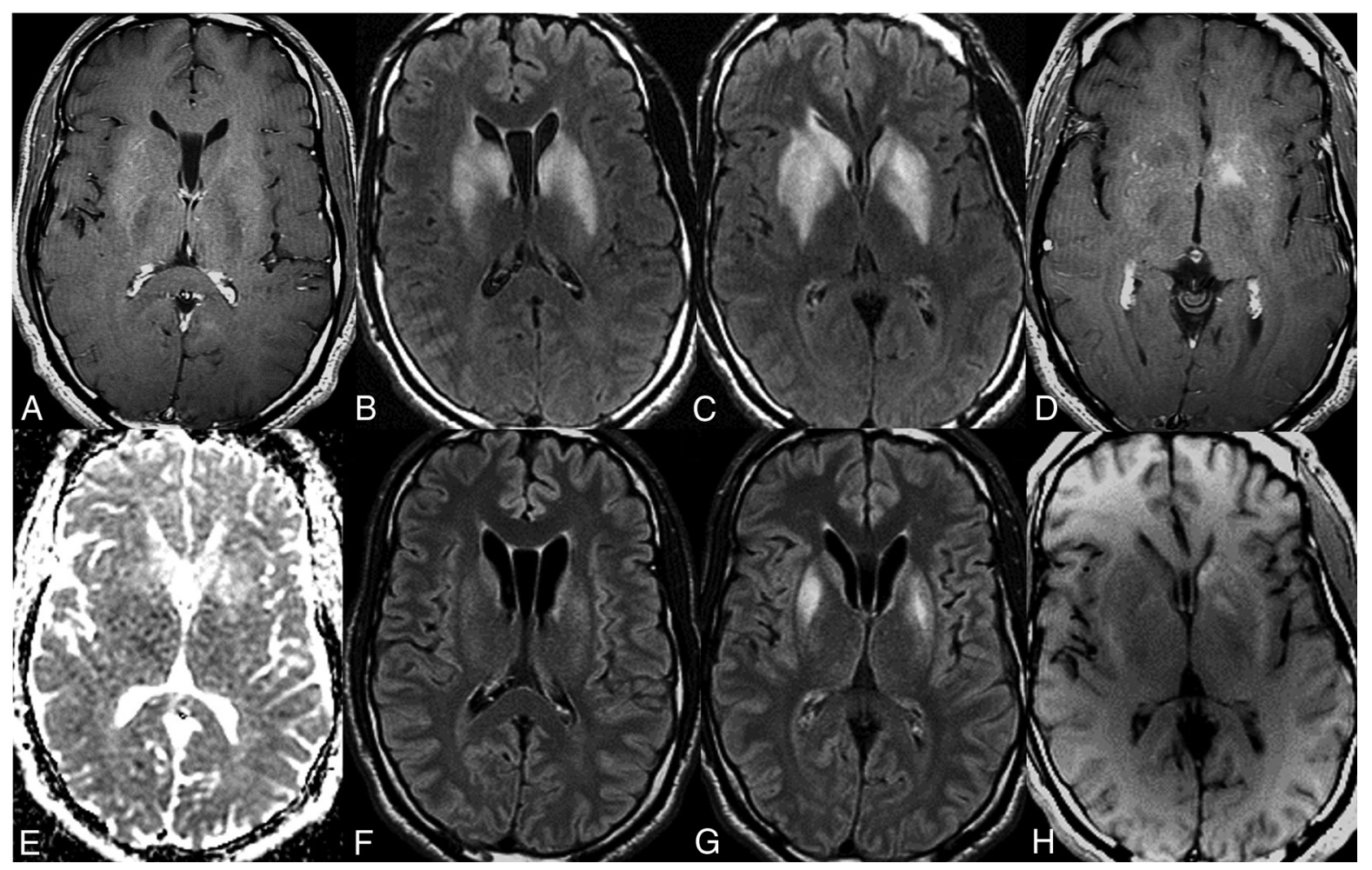

FIG 6. Anti- $N$-methyl-D-aspartate receptor striatal encephalitis. A 38-year-old man presents with progressively worsening cognitive decline, orofacial dyskinesia, and bilateral upper extremity chorea. MR imaging of the brain demonstrates bilateral symmetric T2/FLAIR hyperintensity of the dorsal striatum (caudate and lentiform nucleus) $(B$ and $C$ ) without restricted diffusion $(E)$ or postcontrast enhancement $(A)$. The patient was treated with 3-day IV pulse steroids and 5 rounds of plasmapheresis with a positive clinical response. Follow-up MR imaging of the brain demonstrates reduced T2/FLAIR signal abnormality with atrophy of the caudate heads $(F$ and $G)$. Note the development of intrinsic T1 hyperintensity consistent with coagulative necrosis in the left striatum $(D)$, which persists on follow-up $(H)$. 
fluid is not sufficient alone to establish a particular diagnosis. ${ }^{10,20}$ Antinuclear antibodies such as anti-dsDNA are seen in most patients with SLE and may exist in the absence of neuropsychiatric symptoms. ${ }^{1,24}$ The nonspecific nature of these circulating antibodies is further emphasized by studies demonstrating that various autoantibodies associated with lupus and autoimmune encephalitis have even been reported in asymptomatic, healthy volunteers. ${ }^{17,24}$ The significance of these antibodies in the absence of disease remains unclear, but the identification of specific circulating antibodies in the appropriate clinical context can support the diagnosis and subsequent treatment of an antibody-mediated disorder in patients with unexplained neurologic dysfunction. ${ }^{17,20}$

\section{CONCLUSIONS}

Antibody-mediated diseases are complex and can occur anywhere in the body where the immune system is able to gain access to a target antigen. We believe that striatal-predominant CNS involvement of lupus may represent an under-recognized entity in the general category of NPSLE with features of autoimmune encephalitis, which include similar MR imaging findings and a similar therapeutic response to early plasmapheresis. The characteristic MRI findings of bilateral symmetric basal ganglionic T2/FLAIR hyperintensity without restricted diffusion or postcontrast enhancement are quite striking, and although these imaging findings are non-specific, many of the other possible etiologies can be excluded during the diagnostic workup. Neuroimaging has 4 important roles in this setting: excluding more common etiologies, demonstrating findings consistent with an underlying autoimmune process, monitoring the response to therapy (ie, reduction in T2/FLAIR hyperintensity), and identifying complications of the disease (ie, brain atrophy or intrinsic basal ganglionic T1 hyperintensity, possibly reflecting coagulative necrosis) that will impact the long-term prognosis after an episode of antibody-mediated neuroinflammation. Radiologists can have a tremendous impact if they are familiar with and recognize these kinds of antibody-mediated diseases in their practice.

\section{REFERENCES}

1. Borchers AT, Naguwa SM, Shoenfeld Y, et al. The geoepidemiology of systemic lupus erythematosus. Autoimmun Rev 2010;9:A277-87 CrossRef Medline

2. Popescu A, Kao AH. Neuropsychiatric systemic lupus erythematosus. Curr Neuropharmacol 2011;9:449-57 CrossRef Medline

3. Kivity S, Agmon-Levin N, Zandman-Goddard G, et al. Neuropsychiatric lupus: a mosaic of clinical presentations. BMC Med 2015;13:43 CrossRef Medline

4. Gerosa M, Poletti B, Pregnolato F, et al. Antiglutamate receptor antibodies and cognitive impairment in primary antiphospholipid syndrome and systemic lupus erythematosus. Front Immunol 2016; 7:5 CrossRef Medline
5. Kowal C, Degiorgio LA, Lee JY, et al. Human lupus autoantibodies against NMDA receptors mediate cognitive impairment. Proc Natl Acad Sci U S A 2006;103:19854-59 CrossRef Medline

6. Brimberg L, Mader S, Fujieda Y, et al. Antibodies as mediators of brain pathology. Trends Immunol 2015;36:709-24 CrossRef Medline

7. DeGiorgio LA, Konstantinov KN, Lee SC, et al. A subset of lupus antiDNA antibodies cross-reacts with the NR2 glutamate receptor in systemic lupus erythematosus. Nat Med 2001;7:1189-93 CrossRef Medline

8. Gelb S, Stock AD, Anzi S, et al. Mechanisms of neuropsychiatric lupus: the relative roles of the blood-cerebrospinal fluid barrier versus blood-brain barrier. J Autoimmun 2018;91:34-44 CrossRef Medline

9. Küppenbender KD, Standaert DG, Feuerstein TJ, et al. Expression of NMDA receptor subunit mRNAs in neurochemically identified projection and interneurons in the human striatum. J Comp Neurol 2000;419:407-21 CrossRef Medline

10. Dalmau J, Graus F. Antibody-mediated encephalitis. N Engl J Med 2018;378:840-51 CrossRef Medline

11. Dalmau J, Tüzün E, Wu HY, et al. Paraneoplastic anti-N-methyl-Daspartate receptor encephalitis associated with ovarian teratoma. Ann Neurol 2007;61:25-36 CrossRef Medline

12. Kelley BP, Patel SC, Marin HL, et al. Autoimmune encephalitis: pathophysiology and imaging review of an overlooked diagnosis. AJNR Am J Neuroradiol 2017;38:1070-78 CrossRef Medline

13. Dalmau J, Gleichman AJ, Hughes EG, et al. Anti-NMDA-receptor encephalitis: case series and analysis of the effects of antibodies. Lancet Neurol 2008;7:1091-98 CrossRef Medline

14. Luyendijk J, Steens SC, Ouwendijk WJ, et al. Neuropsychiatric systemic lupus erythematosus: lessons learned from magnetic resonance imaging. Arthritis Rheum 2011;63:722-32 CrossRef Medline

15. Rubin DB, Batra A, Vaitkevicius H, et al. Autoimmune neurologic disorders. Am J Med 2018;131:226-36 CrossRef Medline

16. Lee WJ, Lee ST, Byun JI, et al. Rituximab treatment for autoimmune limbic encephalitis in an institutional cohort. Neurology 2016;86: 1683-91 CrossRef Medline

17. Lancaster $E$. The diagnosis and treatment of autoimmune encephalitis. J Clin Neurol 2016;12:1-13 CrossRef Medline

18. Ramos-Casals M, Soto MJ, Cuadrado MJ, et al. Rituximab in systemic lupus erythematosus: a systematic review of off-label use in 188 cases. Lupus 2009;18:767-76 CrossRef Medline

19. Tobin WO, Strand EA, Clark HM, et al. NMDA receptor encephalitis causing reversible caudate changes on MRI and PET imaging. Neurol Clin Pract 2014;4:470-73 CrossRef Medline

20. Graus F, Titulaer MJ, Balu R, et al. A clinical approach to diagnosis of autoimmune encephalitis. Lancet Neurol 2016;15:391-404 CrossRef Medline

21. Dale RC, Church AJ, Surtees RA, et al. Encephalitis lethargica syndrome: 20 new cases and evidence of basal ganglia autoimmunity. Brain 2004;127:21-33 CrossRef Medline

22. Brami-Zylberberg F, Méary E, Oppenheim C, et al. Abnormalities of the basal ganglia and thalami in adults [in French]. J Radiol 2005;86: 281-93 CrossRef Medline

23. Anderson JC, Costantino MM, Stratford T. Basal ganglia: anatomy, pathology, and imaging characteristics. Curr Probl Diagn Radiol 2004;33:28-41 CrossRef Medline

24. Menon S, Jameson-Shortall E, Newman SP, et al. A longitudinal study of anticardiolipin antibody levels and cognitive functioning in systemic lupus erythematosus. Arthritis Rheum 1999;42:735-41 CrossRef Medline 